\title{
Erratum: supersymmetric monojets at the Large Hadron Collider
}

\author{
Benjamin C. Allanach, ${ }^{a}$ Sebastian $\mathrm{Grab}^{b}$ and Howard E. Haber ${ }^{b}$ \\ ${ }^{a}$ DAMTP, CMS, University of Cambridge, \\ Wilberforce Road, Cambridge, CB3 0WA, U.K. \\ ${ }^{b}$ Department of Physics and SCIPP, University of California, \\ Santa Cruz, CA 95064, U.S.A. \\ E-mail: b.c.allanach@damtp.cam.ac.uk, sgrab@scipp.ucsc.edu, \\ haber@scipp.ucsc.edu
}

ERRATUM TO: JHEP01(2011)138

ABSTRACT: Supersymmetric monojets may be produced at the Large Hadron Collider by the process $q g \rightarrow \widetilde{q} \widetilde{\chi}_{1}^{0} \rightarrow q \widetilde{\chi}_{1}^{0} \widetilde{\chi}_{1}^{0}$, leading to a jet recoiling against missing transverse momentum. We discuss the feasibility and utility of the supersymmetric monojet signal. In particular, we examine the possible precision with which one can ascertain the $\widetilde{\chi}_{1}^{0} \widetilde{q} q$ coupling via the rate for monojet events. Such a coupling contains information on the composition of the $\widetilde{\chi}_{1}^{0}$ and helps bound dark matter direct detection cross-sections and the dark matter relic density of the $\widetilde{\chi}_{1}^{0}$. It also provides a check of the supersymmetric relation between gauge couplings and gaugino-quark-squark couplings.

KeYwORDS: Supersymmetry Phenomenology

The left hand side of eq. (A.47) should be $\left|C_{1}(s, t)\right|^{2}$ rather than $C_{1}(s, t)$. Therefore, the correct version of eq. (A.47) is

$$
\left|C_{1}(s, t)\right|^{2}=N\left[\frac{s+t-M^{2}}{2 s}-\frac{M^{2}\left(m^{2}-t\right)}{\left(M^{2}-t\right)^{2}}+\frac{s m^{2}+\left(m^{2}-t\right)\left(M^{2}-m^{2}\right)}{s\left(M^{2}-t\right)}\right] .
$$

